Supporting Information

\title{
Structure and Catalytic Activity of the Gold Clusters \\ Supported on Nitrogen-Doped Graphene
}

\author{
Jing Li, Xiaomei Zhao, Zhongyun Ma*, Yong Pei* \\ ${ }^{a}$ Department of Chemistry, Key Laboratory of Environmentally Friendly Chemistry and Applications of Ministry of \\ Education, Xiangtan University, Xiangtan, 411105, Hunan Province, P.R, China.
}

Figure S1. The relationship of the different box $(a \times b \times c, a=b, c=40 \AA)$ of graphene (randomly doped $12 \mathrm{~N}$ atoms) cell and energy.

Figure S2. The position of the $\mathrm{C}$ atoms substituted by $\mathrm{N}$ atoms around the $\mathrm{C}$ atom (combined with the Au cluster, green atom).

Table S1. Structures and calculated adsorption energies $\left(E_{\mathrm{ads}}, \mathrm{eV}\right)$, net charges $(Q, e)$ and the corresponding Hirshfeld charge analysis of $\mathrm{Au}_{28}$ supported on the graphitic $\mathrm{N}$-doped graphene $\left(\mathrm{G}_{4 \mathrm{~N}}, \mathrm{G}_{10 \mathrm{~N}}, \mathrm{G}_{12 \mathrm{~N}}, \mathrm{G}_{14 \mathrm{~N}}, \mathrm{G}_{16 \mathrm{~N}}, \mathrm{G}_{17 \mathrm{~N}}, \mathrm{G}_{18 \mathrm{~N}}, \mathrm{G}_{2 l \mathrm{~N}}\right)$.

Table S2. The charge state of $\mathrm{Au}_{28} / \mathrm{G}_{22 \mathrm{~N}}$ or $\mathrm{Au}_{28} / \mathrm{G}_{9 \mathrm{~N}, 9 \mathrm{~V}}$ before and after the adsorption of the $\mathrm{O}_{2}$ on the best adsorption site.

Table S3. Structures and adsorption energies of $\mathrm{CO}$ molecules adsorption on $\mathrm{Au}_{28} / \mathrm{G}_{22 \mathrm{~N}}$ and $\mathrm{Au}_{28} / \mathrm{G}_{9 \mathrm{~N}, 9 \mathrm{~V} \text {. }}$ 

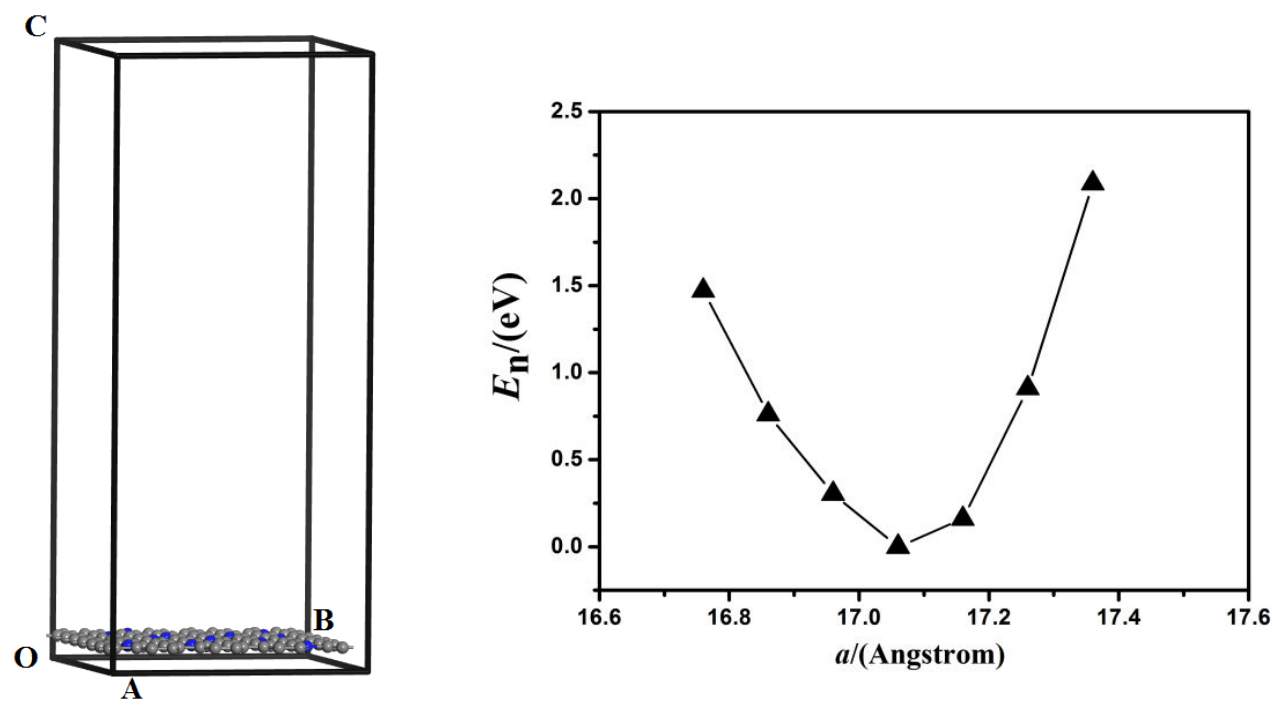

Figure S1. The relationship of the different box $(a \times b \times c, a=b, c=40 \AA)$ of graphene (randomly doped $12 \mathrm{~N}$ atoms) cell and energy. The lowest $E_{\mathrm{n}}$ is set to $0 \mathrm{eV}$.

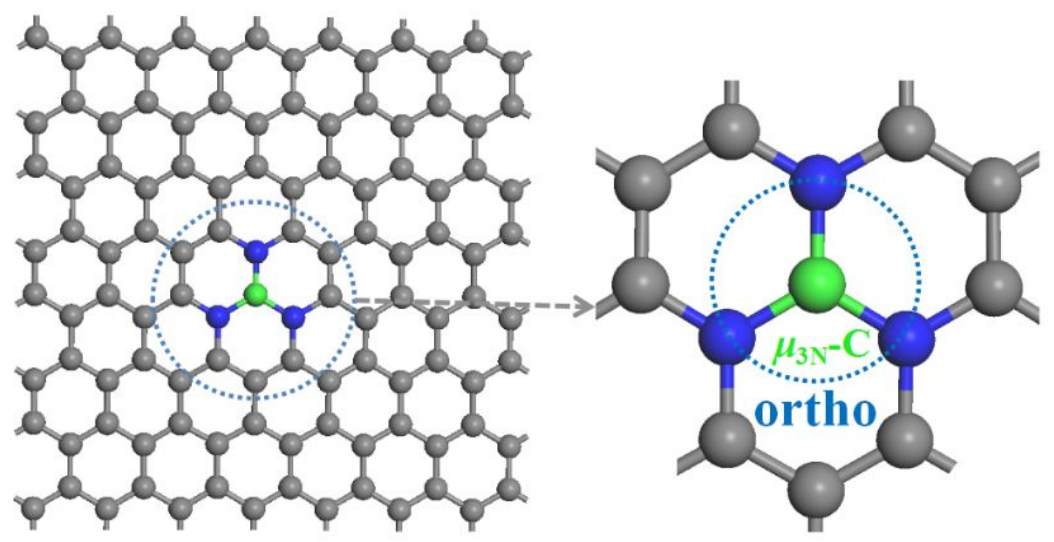

Figure S2. The position of the $\mathrm{C}$ atoms substituted around the $\mathrm{C}$ atom (combined with the Au cluster, green atom). 
Table S1. Structures and calculated adsorption energies $\left(E_{\mathrm{ads}}, \mathrm{eV}\right)$, net charges $(Q, e)$ and the corresponding Hirshfeld charge analysis of $\mathrm{Au}_{28}$ supported on the graphitic N-doped graphene $\left(\mathrm{G}_{4 \mathrm{~N}}, \mathrm{G}_{10 \mathrm{~N}}, \mathrm{G}_{12 \mathrm{~N}}, \mathrm{G}_{14 \mathrm{~N}}, \mathrm{G}_{16 \mathrm{~N}}, \mathrm{G}_{17 \mathrm{~N}}, \mathrm{G}_{18 \mathrm{~N}}, \mathrm{G}_{21 \mathrm{~N}}\right)$. Color scheme: negatively charged $\mathrm{Au}$ atom, blue; positively charged $\mathrm{Au}$ atom, purple; $\mathrm{C}$ atom in graphene, grey; $\mathrm{N}$ atom, blue; $\mathrm{C}$ atoms bind with $\mathrm{Au}$, green.

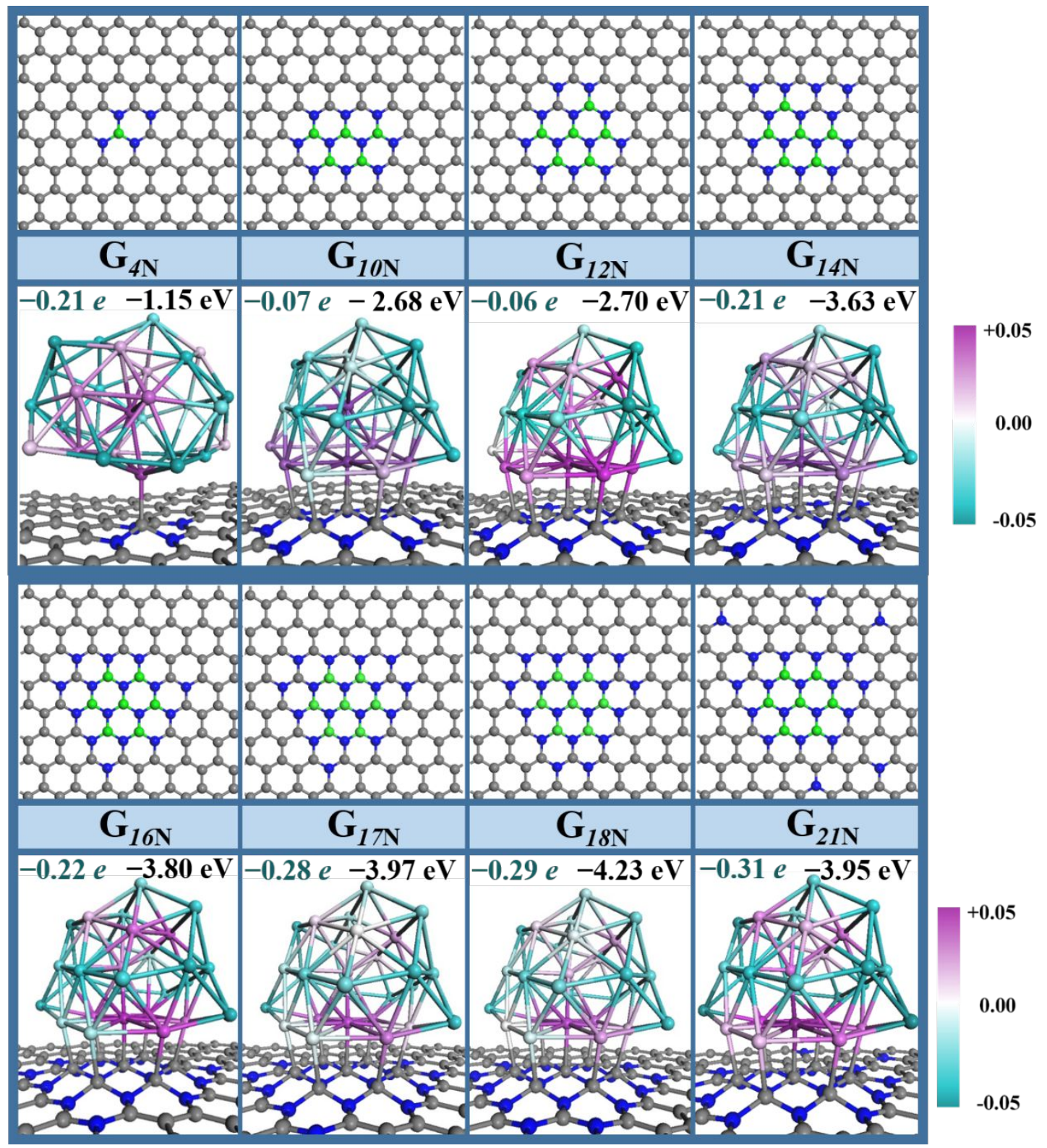


Table S2. The charge state of $\mathrm{Au}_{28} / \mathrm{G}_{22 \mathrm{~N}}$ or $\mathrm{Au}_{28} / \mathrm{G}_{9 \mathrm{~N}, 9 \mathrm{~V}}$ before and after the adsorption of the $\mathrm{O}_{2}$ on the best adsorption site (e).

\begin{tabular}{|c|c|c|c|c|c|c|c|}
\hline $\mathrm{Au}_{28} / \mathbf{G}_{22 \mathrm{~N}}{ }^{a}$ & charge $^{b}$ & charge $2^{c}$ & $\Delta$ charge & $\mathbf{A u}_{28} / \mathbf{G}_{9 \mathrm{~N},}, \mathbf{v v}^{d}$ & charge $^{e}$ & charge $2^{f}$ & $\Delta$ charge \\
\hline $\mathrm{Au}_{(1)}$ & -0.0417 & -0.0496 & -0.0079 & $\mathrm{Au}_{(1)}$ & 0.0072 & 0.0126 & 0.0054 \\
\hline $\mathrm{Au}_{(2)}$ & 0.01 & 0.0078 & -0.0022 & $\mathrm{Au}_{(2)}$ & 0.0429 & 0.0536 & 0.0107 \\
\hline $\mathrm{Au}_{(3)}$ & 0.0035 & 0.0061 & 0.0026 & $\mathrm{Au}_{(3)}$ & -0.0094 & -0.0022 & 0.0072 \\
\hline $\mathrm{Au}_{(4)}$ & -0.0368 & -0.0302 & 0.0066 & $\mathrm{Au}_{(4)}$ & -0.0298 & -0.0349 & -0.0051 \\
\hline $\mathrm{Au}_{(5)}$ & 0.0132 & 0.017 & 0.0038 & $\mathrm{Au}_{(5)}$ & 0.0106 & 0.0048 & -0.0058 \\
\hline $\mathrm{Au}_{(6)}$ & 0.0127 & 0.012 & -0.0007 & $\mathrm{Au}_{(6)}$ & 0.0071 & -0.0112 & -0.0183 \\
\hline $\mathrm{Au}_{(7)}$ & -0.0111 & 0.0498 & 0.0609 & $\mathrm{Au}_{(7)}$ & -0.0152 & 0.0466 & 0.0618 \\
\hline $\mathrm{Au}_{(8)}$ & -0.0206 & -0.0175 & 0.0031 & $\mathrm{Au}_{(8)}$ & 0.0007 & -0.0097 & -0.0104 \\
\hline $\mathrm{Au}_{(9)}$ & -0.0434 & -0.0484 & -0.005 & $\mathrm{Au}_{(9)}$ & -0.0153 & -0.0096 & 0.0057 \\
\hline $\mathrm{Au}_{(10)}$ & 0.0215 & 0.0206 & -0.0009 & $\mathrm{Au}_{(10)}$ & 0.1069 & 0.1185 & 0.0116 \\
\hline $\mathrm{Au}_{(11)}$ & 0.0403 & 0.0451 & 0.0048 & $\mathrm{Au}_{(11)}$ & 0.1135 & 0.1194 & 0.0059 \\
\hline $\mathrm{Au}_{(12)}$ & -0.0156 & -0.0116 & 0.004 & $\mathrm{Au}_{(12)}$ & -0.0152 & -0.0021 & 0.0131 \\
\hline $\mathrm{Au}_{(13)}$ & 0.0263 & 0.026 & -0.0003 & $\mathrm{Au}_{(13)}$ & 0.0665 & 0.0593 & -0.0072 \\
\hline $\mathrm{Au}_{(14)}$ & -0.0401 & -0.0424 & -0.0023 & $\mathrm{Au}_{(14)}$ & -0.0146 & -0.0111 & 0.0035 \\
\hline $\mathrm{Au}_{(15)}$ & -0.0104 & 0.0001 & 0.0105 & $\mathrm{Au}_{(15)}$ & -0.0102 & 0.0642 & 0.0744 \\
\hline $\mathrm{Au}_{(16)}$ & -0.0287 & -0.0307 & -0.002 & $\mathrm{Au}_{(16)}$ & 0.0046 & 0.0184 & 0.0138 \\
\hline $\mathrm{Au}_{(17)}$ & -0.0304 & -0.0297 & 0.0007 & $\mathrm{Au}_{(17)}$ & 0.0035 & 0.0036 & 0.0001 \\
\hline $\mathrm{Au}_{(18)}$ & -0.0248 & -0.0224 & 0.0024 & $\mathrm{Au}_{(18)}$ & -0.0282 & -0.0337 & -0.0055 \\
\hline $\mathrm{Au}_{(19)}$ & 0.0016 & -0.0007 & -0.0023 & $\mathrm{Au}_{(19)}$ & 0.0118 & 0.0148 & 0.0030 \\
\hline $\mathrm{Au}_{(20)}$ & -0.003 & 0.0039 & 0.0069 & $\mathrm{Au}_{(20)}$ & 0.1370 & 0.1386 & 0.0016 \\
\hline $\mathrm{Au}_{(21)}$ & -0.0272 & -0.0225 & 0.0047 & $\mathrm{Au}_{(21)}$ & -0.0036 & 0.0063 & 0.0099 \\
\hline $\mathrm{Au}_{(22)}$ & -0.0431 & -0.0564 & -0.0133 & $\mathrm{Au}_{(22)}$ & 0.1315 & 0.1309 & -0.0006 \\
\hline $\mathrm{Au}_{(23)}$ & -0.0235 & 0.0517 & 0.0752 & $\mathrm{Au}_{(23)}$ & 0.0046 & 0.0330 & 0.0284 \\
\hline $\mathrm{Au}_{(24)}$ & -0.0012 & 0.0174 & 0.0186 & $\mathrm{Au}_{(24)}$ & -0.0091 & 0.0137 & 0.0228 \\
\hline $\mathrm{Au}_{(25)}$ & -0.0184 & -0.0236 & -0.0052 & $\mathrm{Au}_{(25)}$ & 0.0018 & 0.0042 & 0.0024 \\
\hline $\mathrm{Au}_{(26)}$ & 0.0122 & 0.0282 & 0.016 & $\mathrm{Au}_{(26)}$ & 0.0070 & 0.0002 & -0.0068 \\
\hline $\mathrm{Au}_{(27)}$ & -0.0438 & -0.0411 & 0.0027 & $\mathrm{Au}_{(27)}$ & -0.0254 & 0.0181 & 0.0435 \\
\hline $\mathrm{Au}_{(28)}$ & 0.0016 & -0.0024 & -0.004 & $\mathrm{Au}_{(28)}$ & 0.1070 & 0.1111 & 0.0041 \\
\hline sum & -0.3209 & -0.1435 & 0.1774 & sum & 0.5882 & 0.8574 & 0.2692 \\
\hline
\end{tabular}

${ }^{\mathrm{a}}$ Charge partitioning by Hirshfeld method of the $\mathrm{Au}_{28},{ }^{\mathrm{b}}$ the charge state of $\mathrm{Au}_{28}$ cluster supported on $\mathrm{G}_{22 \mathrm{~N}},{ }^{\mathrm{c}}$ the charge state of $\mathrm{Au}_{28}$ cluster supported on $\mathrm{G}_{22 \mathrm{~N}}$ under $\mathrm{O}_{2}$ adsorption on the best adsorption site $\left(\mathrm{Au}_{(7)}-\mathrm{Au}_{(23)}\right) \cdot{ }^{\mathrm{d}}$ Charge partitioning by Hirshfeld method of the $\mathrm{Au}_{28},{ }^{\mathrm{e}}$ the charge state of $\mathrm{Au}_{28}$ cluster supported on $\mathrm{G}_{9 \mathrm{~N}, 9 \mathrm{~V},},{ }^{\mathrm{f}}$ the charge state of $\mathrm{Au}_{28}$ cluster supported on $\mathrm{G}_{9 \mathrm{~N}, 9 \mathrm{~V}}$ under $\mathrm{O}_{2}$ adsorption on the best adsorption site $\left(\mathrm{Au}_{(7)}-\mathrm{Au}_{(15)}\right) . \Delta$ charge $=$ charge2-charge1, sum: the net charge of $\mathrm{Au}_{28}$. 
Table S3. Structures and adsorption energies of $\mathrm{CO}$ molecules adsorption on

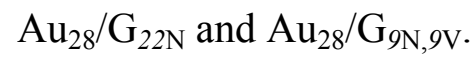

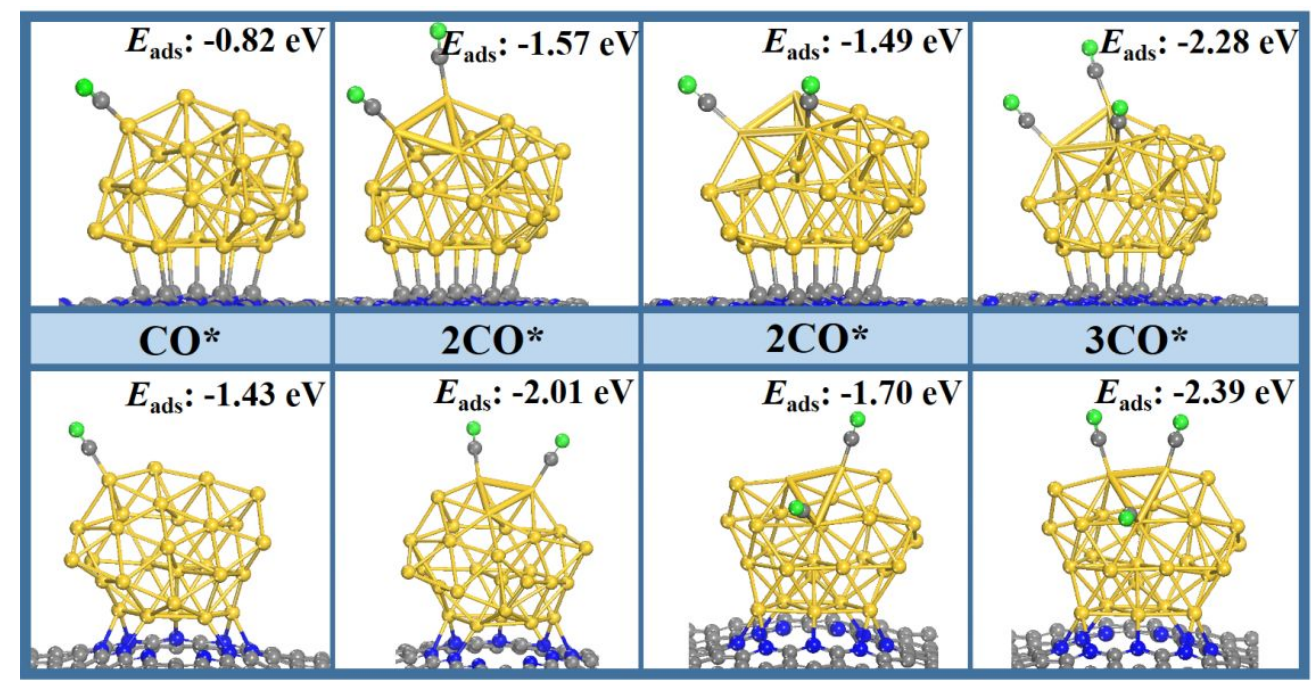

